\title{
PAPEL DE LA ESCUELA Y LOS PROYECTOS DEPORTIVOS DE CENTRO EN EL FOMENTO DE LA PRÁCTICA DE ACTIVIDAD FÍSICA DE LOS ESCOLARES
}

Role of the School and Sports Projects of the Center in Promoting the Practice of Physical Activity Among Schoolchildren

iD Alba Mayor-Villalaín*1

alba.mayor.villalain@uil.es

iD Pedro Cantero-Castrillo²

cantero_46@hotmail.com

\section{Belén Toja-Reboredo}

belen.toja@udc.es

\section{Miguel Ángel González-Valeiro²}

miguel.gonzalez.valeiro@udc.es

* Autor por correspondencia

${ }^{1}$ Universidad Isabel I, España

${ }^{2}$ Universidade da Coruña, España

Fecha recepción: 3/7/2019

Fecha aprobación: 8/11/2019

\section{Resumen}

La legislación educativa en España establece la obligación de adoptar, en los centros educativos, medidas para que la actividad física y la dieta equilibrada formen parte de la población escolar, promoviendo la práctica diaria de actividad física durante la jornada escolar y siguiendo las recomendaciones de los organismos competentes. El profesor de Educación Física es el responsable del diseño, coordinación y supervisión de las medidas que a estos efectos se adopten en el centro educativo. Para ayudar a las instituciones educativas a cumplir con estos requerimientos, en algunas comunidades autónomas, como la gallega, se han creado programas de innovación educativa. Presentamos aquí un estudio descriptivo longitudinal realizado con centros educativos de la comunidad autónoma de Galicia, en el momento inicial de aplicación de dichos programas, denominados Proyectos Deportivos de Centro. Buscábamos realizar esta investigación a modo de diagnóstico para mejorarlos y crear estructuras que en el futuro respondan adecuadamente a la realidad de las escuelas, del profesorado, del alumnado y del entorno (perspectiva ecológica).

Palabras clave: Educación Física, escuela, proyectos deportivos, práctica, práctica de actividad física, secundaria.

\section{Abstract}

The educational legislation in Spain establishes the obligation to adopt in schools, measures to make physical activity and a balanced diet be part of the school population, promoting the daily practice of physical activity during the day and following the recommendations of the competent bodies. The physical education teacher is responsible for the design, coordination and supervision of the measures taken for these purposes in the educational center. To help educational institutions comply with these requirements, in some autonomous communities, such as Galicia, educational innovation programs have been created. We present here a longitudinal descriptive study carried out with educational centers of the autonomous community of Galicia, at the initial moment of application of these programs, called Sports Projects of the Center. We sought to carry out this research as a diagnosis to improve them and create structures that in the future respond adequately to the reality of schools, teachers, students and the environment (ecological perspective).

Keywords: high school, physical activity, Physical Education, school, practice, sports projects. 


\section{MAYOR-VILLALAÍN • CANTERO-CASTRILLO • TOJA-REBOREDO • GONZÁLEZ-VALEIRO}

Papel de la escuela y los proyectos deportivos de centro en el fomento de la práctica...

\section{Introducción}

En los últimos años han sido frecuentes los estudios descriptivos sobre los estilos de vida. En todos se ha constatado un elevado porcentaje de jóvenes que no cumplen las recomendaciones de práctica de actividad física (AF), situación agravada en el grupo de chicas.

La Xunta de Galicia, institución responsable del sistema educativo de la Comunidad Autónoma de Galicia en España, persigue modificar esta realidad a través de una iniciativa destinada a la promoción de estilos de vida activos y saludables, denominados Proyectos Deportivos de Centro (PDC). Estos están enmarcados en el plan Proxecta, que fomenta la innovación educativa a través de programas que desarrollan las competencias como eje del currículum y los elementos transversales.

La importancia de los PDC recae en la apuesta que se realiza para convertir el centro educativo en un espacio dinamizador y promotor de hábitos de vida saludables, tratando de alcanzar una juventud educada físicamente, con una familia, grupo de iguales y entorno implicados en el proceso, en la búsqueda de un mayor impacto de lo que se reserva de forma exclusiva en el currículum, tal como recomiendan autores como Piéron et al. (2007) y Mourelle (2014).

Para orientar a los centros, la Xunta de Galicia da a conocer las experiencias en algunos de los centros participantes y dicta una serie de recomendaciones para llevarlas a cabo, distinguiendo actividades dentro del horario lectivo (dentro y fuera del currículum) y actividades fuera del horario lectivo.

Las primeras recogen recomendaciones como: unidades didácticas transversales; organización de recreos activos; creación de ligas internas; cesión de material; organización de espacios activos con preferencia femenina; utilización de las horas de tutoría para realizar AF; organización de charlas y/o conferencias sobre AF y salud, entre otras. En el caso de las actividades fuera del horario lectivo, dentro del centro educativo o en su entorno, la administración propone: la realización del trayecto a la escuela en bicicleta o caminando; programas de AF mediante las instituciones que gestionan el deporte en edad escolar; programas de actividades de sensibilización a las familias; encuestas de hábitos de AF, entre otras posibilidades. Fuera del horario lectivo y del centro educativo se plantea participar en el + XOGADE y organizar semanas blancas/azules/verdes.

El objetivo del estudio general ha sido más ambicioso, pero en este artículo pretendemos describir los cambios que se producen en la práctica de AF del alumnado de Educación Secundaria Obligatoria (ESO) (12-18 años) de Galicia tras la aplicación de los PDC durante dos cursos académicos.

\section{Revisión de la literatura}

En el 2015, la Organización Mundial de la Salud (OMS) clasifica la inactividad física como el cuarto factor de riesgo en lo que respecta a la mortalidad mundial $(6 \%$ de las muertes registradas en todo el mundo), y determina que las personas que no hacen suficiente AF presentan un riesgo de mortalidad de entre un $20 \%$ y un $30 \%$ superior al de aquellas que son lo suficientemente activas. Vemos como la AF regular presenta beneficios tanto fisiológicos, como psicológicos y sociales sobre la salud (Bermúdez, 2015).

Las preguntas que nos surgen son: ¿Cuál es la cantidad e intensidad diaria de AF recomendada?, y ¿qué tipo de práctica?

Corbin (2014) proponía la llamada Pirámide de AF para adolescentes, mediante la cual da a conocer todo lo necesario sobre el tipo, cantidad e intensidad de AF adecuada en cada momento. Esta fue adaptada posteriormente por el Ministerio de Sanidad y Consumo (Martínez et al., 2008) dentro de AF para la Infancia y Adolescencia, y en 2008 la Agencia Espanoola de Seguridad Alimentaria y Nutrición publicó la Pirámide NAOS (Figura 1), la cual complementa las recomendaciones alimentarias con la necesidad de realizar práctica de $\mathrm{AF}$. 


\section{Figura 1. Pirámide NAOS}

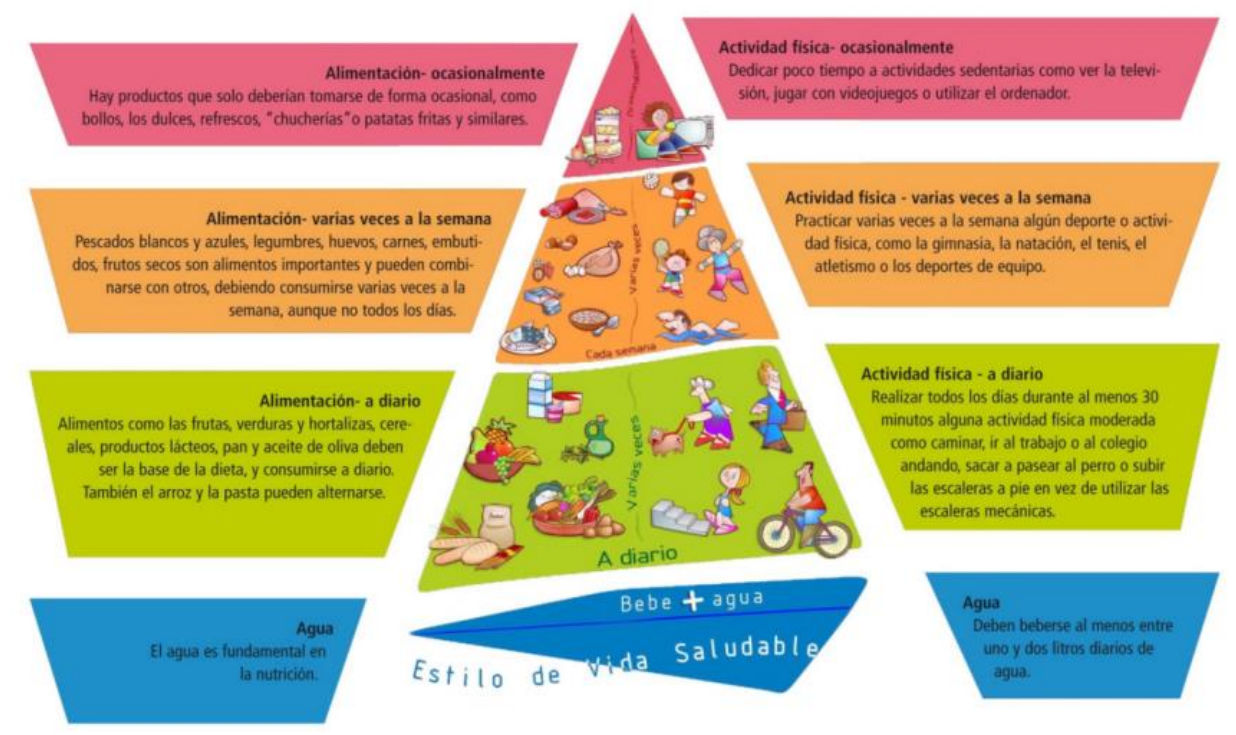

Nota: Ballesteros-Arribas et al. (2008). https://binged.it/2Rsbzm2

En esta pirámide comprobamos como la AF abarca todas las acciones que entrañan movimiento corporal. Aparecen en los primeros peldaños todas las que se pueden realizar como parte de un estilo de vida activo (desplazamientos activos, sacar a pasear al perro, subir las escaleras, momentos de juego, de trabajo, de tareas domésticas...), y a medida que se asciende, esas actividades tienden a dirigirse más hacia el ejercicio físico (gimnasia, natación, zumba, yoga, tenis...), para acabar finalmente recomendando dedicar el menor tiempo posible a las inactivas (ver la televisión, jugar a videojuegos, entrar en Internet...).

En 2016, el Grupo colaborativo de la Sociedad Española de Nutrición Comunitaria añadió a la base de la pirámide la promoción del equilibrio emocional (Figura 2). Según nos indica su guía alimentaria (SENC, 2016), dedicar tiempo a la compra, a la cocción de alimentos y compartir el conocimiento a través de comidas en familia podrían ser los primeros pasos para alcanzar la plena consciencia emocional en el proceso de alimentación. Del mismo modo, la práctica de AF ya no solo se contempla como práctica individual, sino que se potencia su práctica compartida.

\section{Figura 2. Pirámide SENC}

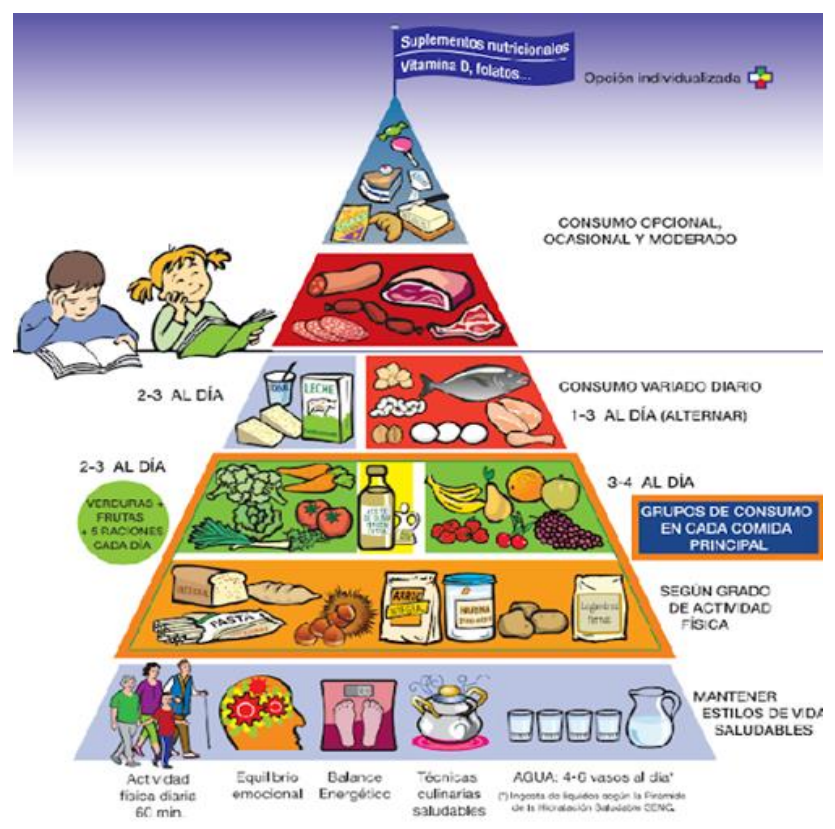

Nota: Grupo Colaborativo de la Sociedad Española de Nutrición Comunitaria, 2016. https://binged.it/2Pp2pEj

En España, el Ministerio de Sanidad, Servicios Sociales e Igualdad realiza las recomendaciones Nacionales de AF para la salud, reducción del sedentaris- 


\section{MAYOR-VILLALAÍN • CANTERO-CASTRILLO • TOJA-REBOREDO • GONZÁLEZ-VALEIRO}

Papel de la escuela y los proyectos deportivos de centro en el fomento de la práctica...

mo y del tiempo de pantalla (2015), las cuales están basadas en las existentes a nivel internacional (OMS, Agencia de Salud Pública de Canadá, Departamento de Salud de EEUU, Sistema Nacional de Salud del Reino Unido, etc.) y se adaptan a la realidad poblacional de nuestro entorno, ya que hasta entonces no existían.

Presentamos brevemente las recomendaciones de la OMS (2010) y su adaptación a la población española para la franja de los cinco a los 17 ańos (edad escolar sobre la que nos centramos en esta investigación), pues ambas se complementan y aportan una visión más completa de manera conjunta. Consiste en juegos, deportes, desplazamientos, actividades recreativas, EF o ejercicios programados, en el contexto de la familia, la escuela o las actividades comunitarias.

Las pautas son:

- Acumular un mínimo de 60 minutos diarios de AF moderada o vigorosa.

- La AF por un tiempo superior a 60 minutos diarios reportará un beneficio aún mayor para la salud.

- La AF diaria debería ser, en su mayor parte, aeróbica. Convendría incorporar, como mínimo tres veces por semana, actividades vigorosas que refuercen, en particular, los músculos y huesos.

- Limitar el tiempo frente a la televisión u otras pantallas (móviles, videojuegos, ordenadores, etc.) con fines recreativos a un máximo de dos horas al día.

- Minimizar el tiempo de transporte motorizado (en coche, en autobús, en metro) y fomentar el transporte activo, recorriendo a pie o en bicicleta al menos parte del camino.

- Fomentar las actividades al aire libre.

Destacamos que, en sus recomendaciones, la OMS (2010) aconseja fomentar un plan de AF inicialmente moderado, que progrese gradualmente hasta alcanzar una mayor intensidad.
La Canadian Society for Exercise Physiology (CSEP/ SCPE) (Tremblay et al., 2016) elabora sus recomendaciones atendiendo a parámetros diarios de práctica de AF, actividades sedentarias y horas de sueño. Afirma que, para obtener beneficios óptimos para la salud, en la infancia y la adolescencia (de cinco a 17 años) se deben alcanzar altos niveles de práctica (al menos 60 minutos diarios de actividad moderada o vigorosa, estás últimas al menos tres días), bajos niveles de comportamiento sedentario y dormir lo suficiente cada día (no menos de ocho horas en ninguna franja de edad).

Interviniendo en la escuela, se puede conseguir llegar a toda la población que está inmersa de forma obligatoria hasta los 16 ańos, y también, a los agentes socializadores que más influencia ejercen sobre los escolares. Además, recoge a la población en el momento ideal de adquisición de hábitos de vida saludables (Morgan et al., 2003), en un entorno favorable para la práctica de AF (Piéron et al., 2008), y los beneficios de un estilo de vida saludable en las primeras etapas de la vida tendrán repercusiones en las posteriores (Piéron et al., 2007).

Podemos concluir que la escuela es reconocida como el lugar más completo y controlado para aplicar programas de promoción de AF con el fin de intervenir en la mejora de los estilos de vida (Melnyk et al., 2007), ya que juega un papel fundamental en la adherencia a la $\mathrm{AF}$ en la infancia y adolescencia (Jago et al., 2009; Martínez-Baena, 2012; Martínez-López et al., 2009; Pérez-López \& Delgado-Fernández, 2007; Piéron et al., 2008; Romero, 2007; Villard et al., 2007) y ayuda a mejorar tanto los resultados en salud física, emocional y social, como los académicos de los estudiantes (St Leger et al., 2010).

\section{Método}

\subsection{Variables de estudio}

Nuestro objeto de estudio se cińe a la relación de los escolares con la práctica de AF. Es por ello que nuestras variables solo atienden a este aspecto, a pesar de que nuestro instrumento incluye otros, como hábitos alimenticios, hábitos de sueño y opiniones. 
Para facilitar su exposición y estudio, agrupamos las variables en dos dimensiones:

I. Datos personales: Centro, Curso y Género. Tales variables no aparecen reseńadas de forma individual, debido a que son las que se utilizan para segmentar los análisis.

II. Hábitos de vida: Práctica de AF informal, de club, extraescolar y el Tiempo total de AF extraído del cálculo del tiempo de actividades informales, de club, extraescolares y 90 minutos de Educación Física (EF) (ya que los estudiantes no se encuentran en AF moderada vigorosa los 120 minutos establecidos por ley). Se establecen dos categorías, siguiendo las recomendaciones de la OMS en 2010 (Activos: siete horas o más; No activos: menos de siete horas).

\subsection{Población y muestra}

La población de estudio está compuesta por el alumnado de 16 centros educativos públicos de la Comunidad Autónoma de Galicia en España, que han aplicado PDC durante los cursos académicos 20132014 y 2014-2015. En el primer momento, fueron 23 centros públicos los que aplicaron PDC, pero se tuvo que descartar a seis de ellos, ya que no continuaron aplicándolos en el segundo momento. Otro centro se utilizó en el estudio piloto.

Para asegurar el número de estudiantes necesarios en representación de la muestra, utilizamos el criterio de seleccionar, por cada cuatro líneas, dos; por cada dos, una de ellas; si el centro solo disponía de una única línea, se seleccionó todo el alumnado de esa aula. En el curso escolar 2013-2014 se recogió información del alumnado de primero, segundo y tercer grado de la ESO. En el curso 2014-2015, se realizó en segundo, tercero y cuarto grado de la ESO, eliminando a primer grado de la ESO e integrando a cuarto, para poder realizar el estudio longitudinal.

En la Tabla 1 se observa la distribución de la muestra en función del género de los escolares en ambos momentos de estudio. Podemos ver en la muestra que el reparto en chicos y chicas es equilibrado (1093 suje- tos en el primer momento y 1289 en el segundo). El número de alumnas en ambos momentos del estudio es un poco superior ( $2 \%$ de media en la diferencia entre ellos).

Tabla 1. Distribución de la muestra en función del género de los escolares

\begin{tabular}{rrrr}
\hline MOMENTO & GÉNERO & N & \% \\
\hline \multirow{2}{*}{$2013-2014$} & MASCULINO & 531 & $\mathbf{4 8 . 6}^{*}$ \\
& FEMENINO & 562 & $\mathbf{5 1 . 4}^{*}$ \\
& TOTAL & 1093 & 100,0 \\
\hline \multirow{2}{*}{$2014-2015$} & MASCULINO & 630 & $\mathbf{4 8 . 9 ^ { * }}$ \\
& FEMENINO & 659 & $\mathbf{5 1 . 1 ^ { * }}$ \\
& TOTAL & 1289 & 100.0 \\
\hline
\end{tabular}

Nota: Elaboración propia.

Para el análisis de los datos se utilizaron distintas agrupaciones que provocaron que la muestra se modificase en cada variable analizada. Además, dependiendo de las variables, la frecuencia también sufrió alteraciones, debido a la posibilidad de que los sujetos no respondiesen a todas las preguntas, o que diesen respuestas erróneas, teniendo que ser anuladas algunas de ellas. Como ejemplo, el caso de los sujetos que han seleccionado más de una opción en preguntas que solo admitían una única respuesta. Por este motivo, en cada tabla de resultados se marca el número del alumnado participante y la agrupación seleccionada.

Destacamos que el número de sujetos de la muestra nos permite establecer con rigor ciertos rasgos de generalización para el conjunto de la población.

\subsection{Instrumento}

Como instrumento de recogida de datos se adaptó el Cuestionario Internacional sobre el Estilo de Vida de los Escolares, validado para su aplicación portuguesa por Marques y Carreriro da Costa (2010) y utilizado en diferentes estudios (Carreiro da Costa \& Marques, 2011). Posteriormente, fue adaptado al gallego y validado por Mourelle (2014). Quedó constituido por 29 preguntas cerradas. Este cuestionario sufrió una nueva adaptación para formar la versión final, con- 


\section{MAYOR-VILLALAÍN • CANTERO-CASTRILLO • TOJA-REBOREDO • GONZÁLEZ-VALEIRO}

Papel de la escuela y los proyectos deportivos de centro en el fomento de la práctica...

formada por 39 ítems y mantuvo la misma estructura que el cuestionario original: cuatro dimensiones, con la siguiente distribución de los ítems: datos personales (6 ítems), hábitos de vida (12 ítems), actitudes y percepciones (12 ítems) y valoración de la escuela, la EF y la práctica de AF (9 ítems).

Los procesos de validación se recogen en Marques y Carreiro da Costa (2013), para escolares portugueses de enseñanza secundaria, Mourelle (2014). En cuanto a la población española, la versión final pasó por un proceso de validación de expertos (Mayor, 2018).

Antes de administrar definitivamente el cuestionario, se realizó un estudio piloto, con una muestra de las mismas características que las de nuestro estudio, al grupo de niños de menor edad, analizándose posteriormente el índice de consistencia interna mediante el Alpha de Cronbach (Tabla 2), con un valor de $\alpha=.78$ para el global del cuestionario.

\subsection{Aspectos éticos y legales}

Para el desarrollo de este proyecto se contó con el conocimiento y el apoyo de la Xunta de Galicia a través de la Secretaría General para el Deporte, que remitió a todos los centros participantes un escrito donde informaba de la investigación y solicitaba su colaboración en la misma.

Inicialmente, el grupo de investigación pasó una invitación para participar a cada uno de los centros, acordando las fechas para su aplicación, y se introdujeron en el estudio aquellos que tenían PDC en el curso escolar 2013-2014 y que los siguieron aplicando en el 2014-2015.

Antes de comenzar el estudio, se pidió el consentimiento informado por parte de la dirección de los centros educativos y el consentimiento por escrito de las familias de los menores, garantizando en todo el proceso el completo anonimato de los participantes.

\section{Resultados}

A continuación, se presenta el análisis comparativo entre la muestra de los estudiantes del 2013-2014 (momento 1) con la muestra del 2014-2015 (mo- mento 2), analizando descriptivamente cada variable y comprobando si estadísticamente se encuentran diferencias significativas entre los dos momentos del estudio.

Los resultados se han organizado en cuatro apartados que se corresponden con las variables estudiadas en el cuestionario, vinculadas a la práctica de $\mathrm{AF}$ del alumnado encuestado, Tiempo de práctica AF informal, deporte de club y extraescolar, finalizando con el Tiempo Total, sumadas todas las variables citadas más el tiempo dedicado a la Práctica de AF formal (EF).

\subsection{Tiempo de práctica semanal informal}

Esta variable se trata en el cuestionario (ítem 10.c) para cuantificar el tiempo que emplea el alumnado en $\mathrm{AF}$ informal de acuerdo con una pregunta anterior de filtro (ítem 10.a). El tiempo de práctica semanal de actividad físico-deportiva informal tiene como posibles respuestas: (1) aproximadamente media hora, (2) aproximadamente una hora, (3) aproximadamente de dos a tres horas, (4) aproximadamente de cuatro a seis horas, (5) siete horas o más.

En la Tabla 2 se observan las frecuencias y porcentajes obtenidos en esta variable, descartando al alumnado que no respondió en la pregunta de filtro y al que respondió nunca ( $11 \%$ del total), coincidente con el porcentaje del alumnado que no ha respondido a esta pregunta. Los mayores porcentajes se distinguen en dos a tres horas por semana en el grupo de chicos ( $35 \%$ de media) en ambos momentos, mientras en el grupo de chicas en una hora ( $42 \%$ de media) en ambos momentos. Los porcentajes disminuyen a medida que nos alejamos tanto por encima como por debajo de una a tres horas en ambos géneros y momentos. Finalmente, podemos concluir que el tiempo de dedicación semanal a las actividades informales, en ambos géneros, tiende a mantenerse e incluso muestra un leve aumento en el segundo momento, ya que en el grupo de chicos se ha incrementado de dos a seis horas semanales $(1 \%)$ y se ha visto reducida la opción de media a una hora semanal (1\%). En el caso de las chicas se ha aumentado de dos a seis horas semanalmente (1\%), para reducir en una hora semanal (1\%). 
Tabla 2. Tiempo de práctica semanal de actividad físico-deportiva informal de todo el alumnado en 2013-14 y 2014-15

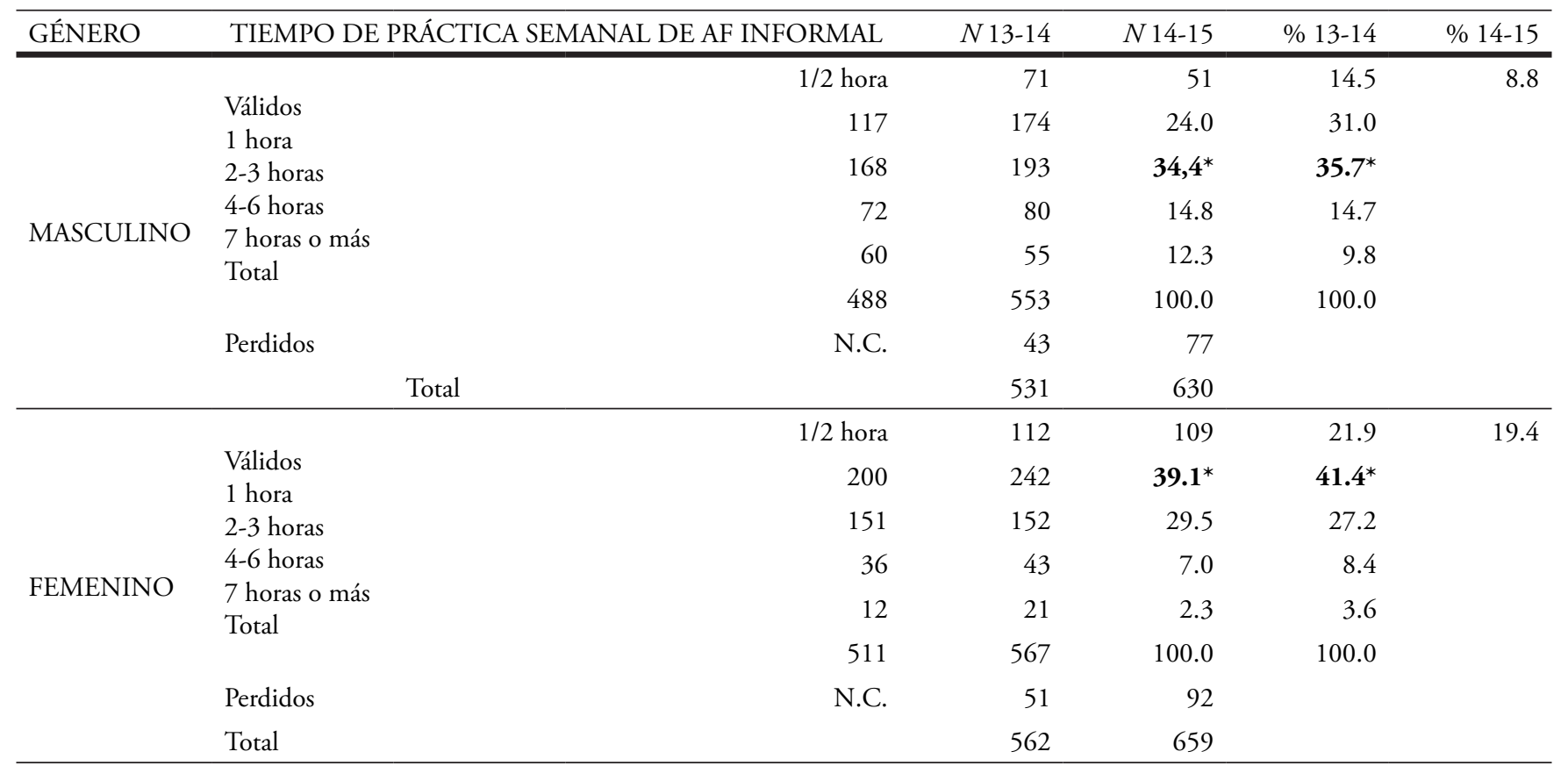

Nota: Elaboración propia.

Como se puede comprobar en la prueba $U$ de Mann-Whitney (Tabla 3) y en la prueba Z de Kolmogorov Smirnov (Tabla 4), tanto la muestra de chicos como la de chicas son iguales en ambos momentos, pues no se presentan diferencias estadísticamente significativas.

Tabla 3. Comparación del tiempo de práctica semanal de actividad físico-deportiva informal de todo el alumnado del 2013-14 con el 2014-15 con la prueba Mann-Whitney

\begin{tabular}{llr}
\hline U de Mann-Whitney: Estadísticos de contraste \\
\hline \multirow{2}{*}{ GÉNERO } & & TIEMPO DE PRÁC- \\
& & $\begin{array}{r}\text { TICA SEMANAL DE } \\
\text { AF INFORMAL }\end{array}$ \\
\hline & U de Mann-Whitney & 133389,500 \\
MASCULINO & $W$ de Wilcoxon & 286570,500 \\
& $Z$ & -.330 \\
& Sig. asintót. (bilateral) & .741 \\
\hline & $U$ de Mann-Whitney & 142141,000 \\
FEMENINO & W de Wilcoxon & -.563 \\
& $Z$ & .573 \\
\hline
\end{tabular}

Nota: Elaboración propia.
Tabla 4. Comparación del tiempo de práctica semanal de actividad físico-deportiva informal de todo el alumnado del 2013-14 con el 2014-15 con la prueba Z de Kolmogorov-Smirnov

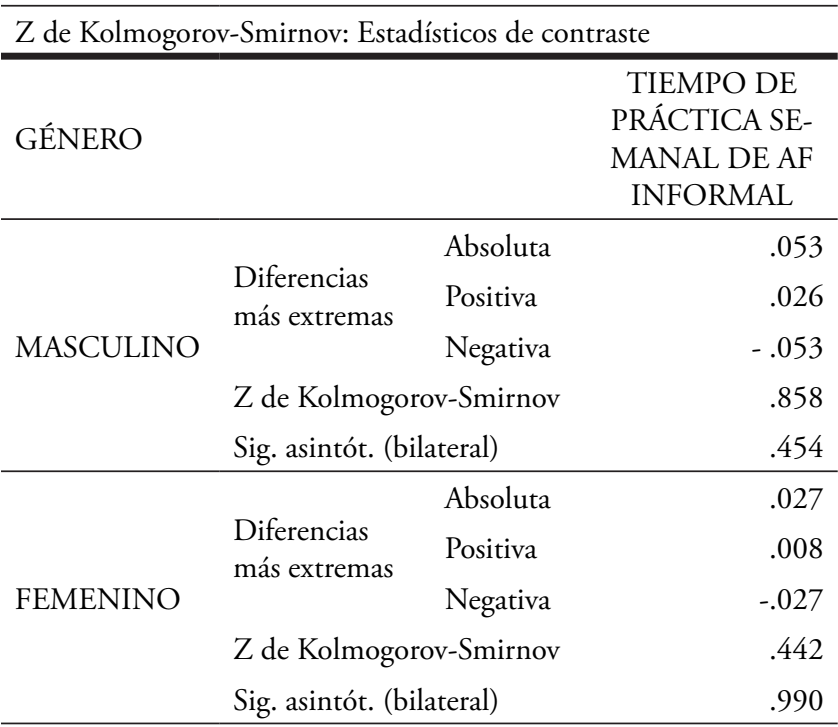

Nota: Elaboración propia.

\subsection{Tiempo de práctica semanal de club}

Esta variable se trata en el cuestionario (ítem 11.c) para cuantificar el tiempo que emplea el alumnado 
en realizar AF de club, de acuerdo con la pregunta filtro anterior (11.a). Presenta como posibles respuestas: (1) aproximadamente media hora, (2) aproximadamente una hora, (3) aproximadamente de dos a tres horas, (4) aproximadamente de cuatro a seis horas, (5) siete horas o más.

En la Tabla 5 se observan las frecuencias y porcentajes obtenidos en esta variable, descartando al alumnado que no respondió en la pregunta de filtro y al que respondió nunca ( $47 \%$ del total), coincidente con el porcentaje de alumnado que no ha respondido a esta pregunta. Los mayores porcentajes se distinguen en dos a tres horas por semana ( $40 \%$ de media) en ambos grupos y momentos. Además, los porcentajes disminuyen a medida que nos alejamos tanto por encima como por debajo de dos a tres horas en ambos géneros y momentos. Finalmente, podemos concluir que el tiempo de práctica semanal de club, en ambos géneros, se ha mantenido en ambos momentos, ya que en el grupo de chicos se ha reducido la opción de media hora y siete horas o más semanalmente $(4 \%)$ y se ha visto incrementado en una hora semanal y de cuatro a seis horas (4\%). En el caso de las chicas se reduce en media hora y dos-tres horas semanales $(6 \%)$, para aumentar en una hora semanal y siete horas o más semanales $(6 \%)$.

Tabla 5. Tiempo de práctica semanal de actividad físico-deportiva de club de todo el alumnado en 201314 y 2014-15

\begin{tabular}{|c|c|c|c|c|c|c|}
\hline GÉNERO & \multicolumn{2}{|c|}{$\begin{array}{l}\text { TIEMPO DE DEDICACIÓN SEMANAL DE } \\
\text { DEPORTE DE CLUB }\end{array}$} & $\begin{array}{c}N \\
13-14 \\
\end{array}$ & $\begin{array}{c}N \\
14-15 \\
\end{array}$ & $\begin{array}{c}\% \\
13-14 \\
\end{array}$ & $\begin{array}{c}\% \\
14-15 \\
\end{array}$ \\
\hline \multirow{8}{*}{ MASCULINO } & \multirow{6}{*}{ Válidos } & $1 / 2$ hora & 21 & 14 & 5.8 & 3.7 \\
\hline & & 1 hora & 46 & 55 & 12.8 & 14.7 \\
\hline & & 2-3 horas & 147 & 156 & $40.9^{*}$ & $41.7^{*}$ \\
\hline & & 4-6 horas & 73 & 83 & 20.3 & 22.2 \\
\hline & & 7 horas o más & 72 & 66 & 20.1 & 17.6 \\
\hline & & Total & 359 & 374 & 100.0 & 100.0 \\
\hline & Perdidos & N.C. & 172 & 256 & & \\
\hline & Total & & 531 & 630 & & \\
\hline \multirow{8}{*}{ FEMENINO } & \multirow{6}{*}{ Válidos } & $1 / 2$ hora & 21 & 11 & 8.4 & 4.2 \\
\hline & & 1 hora & 63 & 75 & 25.1 & 28.8 \\
\hline & & $2-3$ horas & 102 & 98 & $40.6^{*}$ & $37.7^{*}$ \\
\hline & & 4-6 horas & 39 & 41 & 15.5 & 15.8 \\
\hline & & 7 horas o más & 26 & 35 & 10.4 & 13.5 \\
\hline & & Total & 251 & 260 & 100.0 & 100.0 \\
\hline & Perdidos & N.C. & 311 & 399 & & \\
\hline & Total & & 562 & 659 & & \\
\hline
\end{tabular}

Nota: Elaboración propia. 
Como se puede comprobar en la prueba $U$ de Mann-Whitney (Tabla 6) y en la prueba Z de Kolmogorov Smirnov (Tabla 7), tanto la muestra de chicos como la de chicas son iguales en ambos momentos (no se presentan diferencias estadísticamente significativas).

Tabla 6. Comparación del tiempo de práctica semanal de actividad físico-deportiva de club de todo el alumnado del 2013-14 con el 2014-15 con la prueba Mann-Whitney

\begin{tabular}{|c|c|c|}
\hline \multicolumn{3}{|c|}{ U de Mann-Whitney: Estadísticos de contraste } \\
\hline GÉNERO & & $\begin{array}{l}\text { TIEMPO DE } \\
\text { DEDICACIÓN } \\
\text { SEMANAL DE } \\
\text { DEPORTE DE } \\
\text { CLUB }\end{array}$ \\
\hline $\begin{array}{l}\text { MASCULINO } \\
\text { W de Wilcoxon } \\
\text { Z } \\
\text { Sig. asintót. (bilateral) }\end{array}$ & $\begin{array}{l}\text { U de Mann-Whitney } \\
136719,500 \\
-.197 \\
.844\end{array}$ & 66594,500 \\
\hline $\begin{array}{l}\text { FEMENINO } \\
\text { W de Wilcoxon } \\
\text { Z } \\
\text { Sig. asintót. (bilateral) }\end{array}$ & $\begin{array}{l}\text { U de Mann-Whitney } \\
62847,500 \\
-.883 \\
.377\end{array}$ & 31221,500 \\
\hline
\end{tabular}

Nota: Elaboración propia.

Tabla 7. Comparación del tiempo de práctica semanal de actividad físico-deportiva de club de todo el alumnado del 2013-14 con el 2014-15 con la prueba $\mathrm{Z}$ de Kolmogorov-Smirnov

\begin{tabular}{|c|c|c|c|}
\hline \multicolumn{4}{|c|}{ Z de Kolmogorov-Smirnov: Estadísticos de contraste } \\
\hline GÉNERO & & & $\begin{array}{l}\text { TIEMPO } \\
\text { DE DEDI- } \\
\text { CACIÓN SE- } \\
\text { MANAL DE } \\
\text { DEPORTE } \\
\text { DE CLUB }\end{array}$ \\
\hline \multirow{5}{*}{ MASCULINO } & \multirow{3}{*}{$\begin{array}{l}\text { Diferencias más } \\
\text { extremas }\end{array}$} & Absoluta & .024 \\
\hline & & & .024 \\
\hline & & Negativa & -.021 \\
\hline & \multicolumn{2}{|c|}{$Z$ de Kolmogorov-Smirnov } & .326 \\
\hline & \multicolumn{2}{|c|}{ Sig. asintót. (bilateral) } & 1,000 \\
\hline
\end{tabular}

\begin{tabular}{lllr}
\hline \multirow{4}{*}{ FEMENINO } & Diferencias más & Absoluta & .041 \\
& extremas & Positiva & .000 \\
& & Negativa & -.041 \\
& $Z$ de Kolmogorov-Smirnov & .467 \\
& Sig. asintót. (bilateral) & .981 \\
\hline
\end{tabular}

Nota: Elaboración propia.

\subsection{Tiempo de práctica semanal extraescolar}

Esta variable se analiza en el cuestionario (ítem 12.c) para cuantificar el tiempo que emplea el alumnado en AF extraescolar de acuerdo con la pregunta filtro anterior (12.a). El tiempo de práctica semanal de actividad físico-deportiva extraescolar tiene como posibles respuestas: (1) aproximadamente media hora, (2) aproximadamente una hora, (3) aproximadamente de dos a tres horas, (4) aproximadamente de cuatro a seis horas, (5) siete horas o más.

En la Tabla 8 se observan las frecuencias y porcentajes obtenidos en esta variable, descartando al alumnado que no respondió en la pregunta de filtro (12.a) y al que respondió nunca (68\% del total), coincidente con el porcentaje de alumnado que no ha respondido a esta pregunta. Los mayores porcentajes se distinguen en dos a tres horas por semana en el grupo de chicos (34\% de media) en ambos momentos, mientras en el grupo de chicas en una hora $(37 \%$ de media) en ambos momentos. Los porcentajes disminuyen a medida que nos alejamos tanto por encima como por debajo de dos a tres horas en ambos géneros y momentos. Finalmente, podemos concluir que el tiempo de dedicación semanal a las actividades extraescolares ha mostrado un leve aumento en el segundo momento, ya que en el grupo de chicos se ha reducido de media hora semanal $(7 \%)$ y se ha visto incrementado de dos a tres horas semanales (5\%). En el caso de las chicas se reduce en media hora semanal (11\%), para distribuirse en el resto de los tiempos de práctica. 
MAYOR-VILLALAÍN • CANTERO-CASTRILLO • TOJA-REBOREDO • GONZÁLEZ-VALEIRO

Papel de la escuela y los proyectos deportivos de centro en el fomento de la práctica...

Tabla 8. Tiempo de práctica semanal de actividad físico-deportiva extraescolar de todo el alumnado en 2013-14 y 2014-15

\begin{tabular}{|c|c|c|c|c|c|c|}
\hline GÉNERO & \multicolumn{2}{|c|}{$\begin{array}{l}\text { TIEMPO DE DEDICACIÓN SEMANAL } \\
\text { DE AF EXTRAESCOLAR }\end{array}$} & $\begin{array}{c}N \\
13-14 \\
\end{array}$ & $\begin{array}{c}N \\
14-15\end{array}$ & $\begin{array}{c}\% \\
13-14 \\
\end{array}$ & $\begin{array}{c}\% \\
14-15\end{array}$ \\
\hline \multirow{8}{*}{ MASCULINO } & \multirow{6}{*}{ Válidos } & $1 / 2$ hora & 54 & 37 & 23.2 & 16.4 \\
\hline & & 1 hora & 63 & 67 & 27.0 & 29.6 \\
\hline & & 2-3 horas & 73 & 83 & $31.3^{*}$ & $36.7^{*}$ \\
\hline & & 4-6 horas & 24 & 22 & 10.3 & 9.7 \\
\hline & & 7 horas o más & 19 & 17 & 8.2 & 7.5 \\
\hline & & Total & 233 & 226 & 100.0 & 100.0 \\
\hline & Perdidos & N.C. & 298 & 404 & & \\
\hline & Total & & 531 & 630 & & \\
\hline \multirow{8}{*}{ FEMENINO } & \multirow{6}{*}{ Válidos } & $1 / 2$ hora & 48 & 30 & 31.2 & 19.7 \\
\hline & & 1 hora & 52 & 64 & $33.8^{*}$ & 42.1* \\
\hline & & 2-3 horas & 43 & 40 & 27.9 & 26.3 \\
\hline & & 4-6 horas & 9 & 13 & 5.8 & 8.6 \\
\hline & & 7 horas o más & 2 & 5 & 1.3 & 3.3 \\
\hline & & Total & 154 & 152 & 100.0 & 100.0 \\
\hline & Perdidos & N.C. & 408 & 507 & & \\
\hline & Total & & 562 & 659 & & \\
\hline
\end{tabular}

Nota: Elaboración propia.

Como se puede comprobar en la prueba $U$ de Mann-Whitney (Tabla 9) y en la prueba Z de Kolmogorov Smirnov (Tabla 10), tanto la muestra de chicos como la de chicas son iguales en ambos momentos (no se presentan diferencias estadísticamente significativas).
Tabla 9. Comparación del tiempo de práctica semanal de actividad físico-deportiva extraescolar de todo el alumnado del 2013-14 con el 2014-15 con la prueba Mann-Whitney

\begin{tabular}{llr}
\hline U de Mann-Whitney: Estadísticos de contraste \\
\hline \multirow{2}{*}{ GÉNERO } & \multicolumn{2}{c}{ TIEMPO DE DEDICA- } \\
& & $\begin{array}{l}\text { CIÓN SEMANAL DE } \\
\text { AF EXTRAESCOLAR }\end{array}$ \\
\hline \multirow{3}{*}{ MASCULINO } & U de Mann-Whitney & 24946,500 \\
& W de Wilcoxon & 52207,500 \\
& Sig. asintót. (bila- & $-1,010$ \\
& teral) & .313 \\
\hline & U de Mann-Whitney & 10409,500 \\
& W de Wilcoxon & 22344,500 \\
FEMENINO & $-1,755$ \\
& Z & .079 \\
& Sig. asintót. (bila- \\
& teral)
\end{tabular}

Nota: Elaboración propia. 
Tabla 10. Comparación del tiempo de práctica semanal de actividad físico-deportiva extraescolar de todo el alumnado del 2013-14 con el 2014-15 con la prueba $\mathrm{Z}$ de Kolmogorov-Smirnov

\begin{tabular}{|c|c|c|c|}
\hline \multicolumn{4}{|c|}{ Z de Kolmogorov-Smirnov: Estadísticos de contraste } \\
\hline GÉNERO & & & $\begin{array}{l}\text { TIEMPO DE } \\
\text { DEDICACIÓN } \\
\text { SEMANAL } \\
\text { DE AF EX- } \\
\text { TRAESCOLAR }\end{array}$ \\
\hline \multirow{5}{*}{ MASCULINO } & \multirow{3}{*}{$\begin{array}{l}\text { Diferencias } \\
\text { más extremas }\end{array}$} & Absoluta & .068 \\
\hline & & Positiva & .068 \\
\hline & & Negativa & -.012 \\
\hline & \multicolumn{2}{|c|}{ Z de Kolmogorov-Smirnov } & .729 \\
\hline & \multicolumn{2}{|c|}{ Sig. asintót. (bilateral) } & .663 \\
\hline \multirow{5}{*}{ FEMENINO } & \multirow{3}{*}{$\begin{array}{l}\text { Diferencias } \\
\text { más extremas }\end{array}$} & Absoluta & .114 \\
\hline & & Positiva & .114 \\
\hline & & Negativa & .000 \\
\hline & \multicolumn{2}{|c|}{ Z de Kolmogorov-Smirnov } & 1,000 \\
\hline & \multicolumn{2}{|c|}{ Sig. asintót. (bilateral) } & .270 \\
\hline
\end{tabular}

Nota: Elaboración propia.

\subsection{Tiempo total de práctica de actividad física semanal categorizado}

El Tiempo Total de práctica semanal (categorizado) se extrae a partir de la suma de los tiempos de Práctica formal, informal, de club y extraescolar. Consideramos que la $\mathrm{AF}$ formal semanal para el alumnado es de 90 minutos en clase de EF, ya que los estudiantes suelen dedicar diez minutos a los desplazamientos y los cambios de indumentaria de los 120 minutos establecidos por ley.

En este apartado, debido al alto porcentaje de estudiantes no-activos, se incorporan dos categorías con el fin de diferenciar, dentro del grupo de los que son no-activos, los que son poco activos (de cuatro horas a 6.99 horas semanales) de los que son muy poco activos (de cero horas a 3.99 semanalmente) y, del mismo modo, diferenciamos los que son suficiente- mente activos (de siete horas a 9.99 horas semanales), de los que son muy activos (más de diez horas semanales).

Aunque las diferencias no siempre son significativas estadísticamente, los resultados nos muestran que a pesar de la tendencia en la población escolar a reducir la práctica de $\mathrm{AF}$, los proyectos deportivos en los centros estudiados han logrado frenar el acusado descenso que se venía observando, llegando incluso a mantener la práctica del grupo de chicas.

Tal y como se observa en la Tabla 11, más de tres cuartas partes de las alumnas se encuentran por debajo del tiempo de práctica recomendado $(80 \%$ de media en ambos momentos) por la OMS (2010), pero si observamos la diferencia entre momentos podemos ver que hay una tendencia a mantenerse las alumnas activas, y cómo descienden las muy poco activas (2\%), para aumentar las poco activas $(1 \%)$ y muy activas (1\%). En los alumnos, en cambio, vemos cómo el porcentaje de no-activos es menor que en las alumnas, situándose en más de la mitad (58\% de media en ambos momentos). En ellos observamos, además, cómo los porcentajes presentan una tendencia de aumento en los no-activos en el segundo momento (4\%), mientras que, de manera proporcional, los que se encuentran dentro del tiempo de práctica recomendado tienden a disminuir. Ahora bien, dentro de los que no son activos, podemos apreciar cómo los mayores cambios se encuentran en los poco activos (aumento del 3\%), mientras en los activos están en los muy activos (descenso del 3\%). Lo que parece indicarnos que los estudiantes que realizaban mucha práctica de AF semanal van abandonándola, para acabar convirtiéndose en poco activos.

Finalmente, podemos concluir que el tiempo total de práctica de $\mathrm{AF}$ semanal en el grupo de chicas ha tendido hacia un aumento en el segundo momento y en el de chicos a un descenso. 
MAYOR-VILLALAÍN • CANTERO-CASTRILLO • TOJA-REBOREDO • GONZÁLEZ-VALEIRO

Papel de la escuela y los proyectos deportivos de centro en el fomento de la práctica...

Tabla 11. Clasificación con base en la actividad física realizada semanalmente de todo el alumnado en 2013-14 y 2014-15

\begin{tabular}{|c|c|c|c|c|c|c|}
\hline GÉNERO & \multicolumn{2}{|c|}{$\begin{array}{l}\text { TIEMPO TOTAL DE AF CON LA SUMA DE } \\
\text { TODAS LAS VARIABLES }\end{array}$} & \multirow{2}{*}{$\begin{array}{c} \\
13-14 \\
\end{array}$} & \multirow{2}{*}{$\begin{array}{r}\frac{N}{14-15} \\
131\end{array}$} & \multirow{2}{*}{$\begin{array}{c}\begin{array}{c}\% \\
13-14\end{array} \\
21.1\end{array}$} & \multirow{2}{*}{$\begin{array}{c}\begin{array}{c}\% \\
14-15\end{array} \\
21.9\end{array}$} \\
\hline \multirow{5}{*}{ MASCULINO } & \multirow{5}{*}{ Válidos } & Muy poco activo & & & & \\
\hline & & Poco activo & 185 & 225 & $34.8^{*}$ & $37.6^{*}$ \\
\hline & & Activo & 100 & 108 & 18.8 & 18.1 \\
\hline & & Muy activo & 134 & 134 & 25.2 & 22.4 \\
\hline & & Total & 531 & 598 & 100.0 & 100.0 \\
\hline \multirow{5}{*}{ FEMENINO } & \multirow{5}{*}{ Válidos } & Muy poco activo & 268 & 282 & $47.7^{*}$ & 45.9* \\
\hline & & Poco activo & 182 & 204 & 32.4 & 33.2 \\
\hline & & Activo & 67 & 72 & 11.9 & 11.7 \\
\hline & & Muy activo & 45 & 56 & 8.0 & 9.1 \\
\hline & & Total & 562 & 614 & 100.0 & 100.0 \\
\hline
\end{tabular}

Nota: Elaboración propia.

Como se puede comprobar en la prueba $U$ de Mann-Whitney (Tabla 12) y en la prueba Z de Kolmogorov Smirnov (Tabla 13), tanto la muestra de chicos como la de chicas son iguales en ambos momentos (no se presentan diferencias estadísticamente significativas).

Tabla 12. Comparación de la clasificación según el tiempo total de práctica semanal de todo el alumnado del 2013-14 con el 2014-15 con la prueba Mann-Whitney

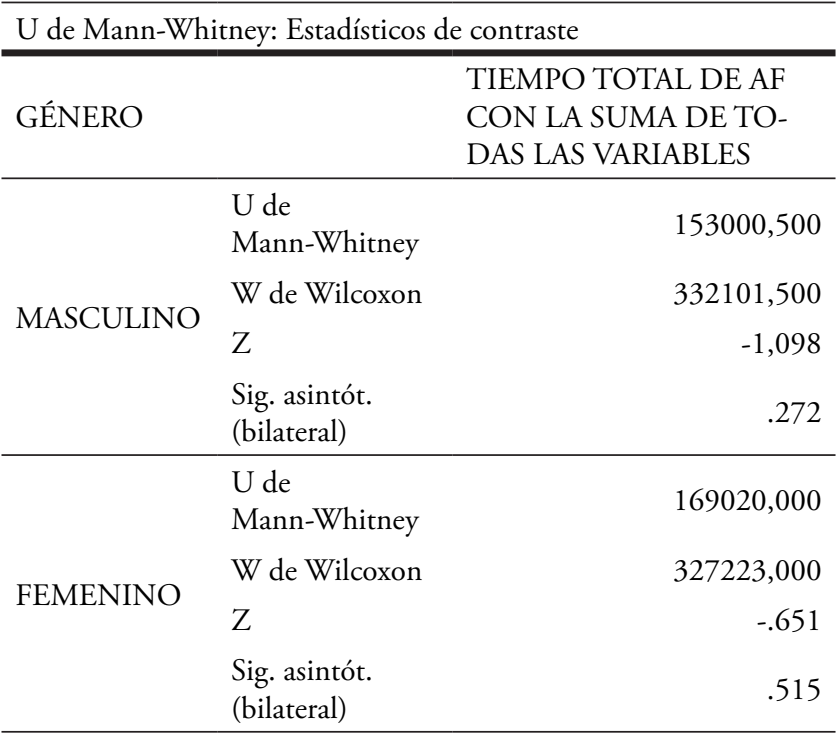

Nota: Elaboración propia.
Tabla 13. Comparación de la clasificación según el tiempo total de práctica semanal de todo el alumnado del 2013-14 con el 2014-15 con la prueba $Z$ de Kolmogorov-Smirnov

\begin{tabular}{|c|c|c|c|}
\hline \multicolumn{4}{|c|}{ Z de Kolmogorov-Smirnov: Estadísticos de contraste } \\
\hline GÉNERO & & & $\begin{array}{l}\text { TIEMPO TO- } \\
\text { TAL DE AF } \\
\text { CON LA SUMA } \\
\text { DE TODAS LAS } \\
\text { VARIABLES }\end{array}$ \\
\hline \multirow{5}{*}{ MASCULINO } & \multirow{3}{*}{$\begin{array}{l}\text { Diferencias } \\
\text { más extremas }\end{array}$} & Absoluta & .036 \\
\hline & & Positiva & .036 \\
\hline & & Negativa & .000 \\
\hline & \multicolumn{2}{|c|}{ Z de Kolmogorov-Smirnov } & .604 \\
\hline & \multicolumn{2}{|c|}{ Sig. asintót. (bilateral) } & .859 \\
\hline \multirow{5}{*}{ FEMENINO } & \multirow{3}{*}{$\begin{array}{l}\text { Diferencias } \\
\text { más extremas }\end{array}$} & Absoluta & .018 \\
\hline & & Positiva & .000 \\
\hline & & Negativa & -.018 \\
\hline & \multicolumn{2}{|c|}{ Z de Kolmogorov-Smirnov } & .301 \\
\hline & \multicolumn{2}{|c|}{ Sig. asintót. (bilateral) } & 1,000 \\
\hline
\end{tabular}

Nota: Elaboración propia. 


\section{Discusión y conclusiones}

Las recomendaciones de la OMS (2010), y su adaptación para la población española (Ministerio de Sanidad, Servicios Sociales e Igualdad, 2015), nos indican cómo entre los cinco y los 17 años se deben acumular un mínimo de 60 minutos diarios de AF moderada o vigorosa. Sin embargo, los nińos y adolescentes no alcanzan los niveles recomendados (Hallal et al., 2012; OMS, 2010; Sallis et al., 2016). De acuerdo con los datos referentes a los 16 centros estudiados, tan solo un $31 \%$ de los escolares cumplen esta recomendación, siendo menor aún el número de chicas que llegan a este mínimo (20\%) que de chicos (42\%).

Comparando los datos con Mourelle (2014), también en población gallega, encontramos que sus resultados recogen un $54 \%$ de escolares activos, lo que refleja un aumento en la inactividad con respecto a los porcentajes observados entonces y cómo se va tendiendo a la situación global descrita por organizaciones como la OMS (2010) y autores como Sallis et al. (2016), donde se encuentran porcentajes muy elevados de adolescentes inactivos. Además, atendiendo a las diferencias respecto al género, constatamos, al igual que nuestros datos, gran diversidad de estudios que reflejan mayor inactividad física en las chicas, entre ellos, el estudio EYHS, con un $9 \%$ de diferencia (Riddoch et al., 2005), estudio HELENA, con un 34\% (Moreno et al., 2008), el estudio HBSC, con un 15\% (Roberts et al., 2009) y el estudio realizado por Mourelle (2014) en escolares gallegos, con un $16 \%$.

El sexo es un correlato que tiene una potente asociación con la participación en AF, pues son las mujeres quienes habitualmente se muestran menos activas que los hombres, entre otros motivos, por las tradicionales tipologías estereotipadas de "hombre físicamente activo" y "mujer físicamente pasiva”, que identifican autores como Crawford y Unger (2004), y que pueden seguir presentes en nuestro contexto.

La secular discriminación que ha sufrido la mujer ha dejado constancia durante dos siglos de un deporte y una EF androcéntricos a los cuales la mujer ha te- nido que adaptarse. Esta situación ha ido evolucionando legislativamente hacia una igualdad real en el contexto educativo, procurando dejar atrás una materia que, según autores como Vázquez et al. (2000), se constituía en un reforzador de estereotipos sobre el propio cuerpo y la AF, así como de las expectativas que a partir de ellos se generan para su futura relación con la AF y el deporte.

No obstante, un análisis del currículum oculto que se está transmitiendo puede ayudar a identificar algunos procedimientos inconscientes que el profesorado y profesionales del contexto deportivo pueden estar retransmitiendo al alumnado. Del mismo modo, el conocimiento e implementación de metodologías de aprendizaje comprensivo pueden contribuir a disminuir las diferencias en la práctica de AF según el género, pues como seńala el Real Decreto 1105/2014, por el que se establece el currículo básico de la Educación Secundaria Obligatoria y del Bachillerato en Espańa, no es suficiente con la mera práctica; la comprensión de los parámetros estructurales y funcionales, o la transferencia entre deportes, lograrán que tanto los chicos como las chicas entiendan el deporte como un recurso para ocupar su tiempo libre de forma saludable.

$\mathrm{Al}$ analizar las diferencias entre momentos, no se encuentran diferencias significativas en el total de práctica semanal de los 16 centros estudiados $(p=.272$ en los chicos y .515 en las chicas), al contrario de lo que ocurre con la tendencia general, donde el porcentaje de alumnado inactivo sigue en un aumento aparentemente imparable (Ruiz-Pérez et al., 2014), la aplicación de los PDC no logró aumentar la práctica de AF, pero consiguió frenar el descenso que se venía auspiciando.

Los datos analizados provienen de la suma de los tiempos de práctica informal, de club y extraescolares de los jóvenes. Se puede afirmar que, atendiendo a las indicaciones de la OMS (2010), a su adaptación para la población española (Ministerio de Sanidad, Servicios Sociales e Igualdad, 2015), junto con la Agencia Española de Seguridad Alimentaria y Nutrición (2008) y CSEP/SCPE (2016), que re- 


\section{MAYOR-VILLALAÍN • CANTERO-CASTRILLO • TOJA-REBOREDO • GONZÁLEZ-VALEIRO}

Papel de la escuela y los proyectos deportivos de centro en el fomento de la práctica...

comiendan para la adolescencia realizar un mínimo de tres veces por semana algún deporte o $\mathrm{AF}$ con una intensidad moderada o vigorosa (ya sea informal o no formal), sólo una pequeña parte del alumnado parece estar cumpliendo dichas recomendaciones.

Gracias al análisis descriptivo de las frecuencias y tiempos de práctica en actividades informales, sabemos que, en el total de los centros, tanto las alumnas como los alumnos se caracterizan por realizar $\mathrm{AF}$ de dos a tres veces por semana $($ chicos $=33 \%$ y chicas $=34 \%)$, con una duración semanal de entre dos y tres horas en los chicos (35\%) y una hora en las chicas (42\%).

Comparando los datos, encontramos similitudes con los recogidos por Marques (2010) en escolares portugueses, pues las mayores frecuencias las sitúa de dos a tres veces por semana (26\%), lo que coincide también con el estudio de la Fundación para la Investigación Nutricional (2016) en escolares españoles. Además, Nuviala et al. (2011) vuelven a coincidir con nuestros datos, encontrando en escolares espańoles diferencias significativas respecto al género.

Al observar la evolución, no se encuentran diferencias significativas ni en la práctica de AF informal de los chicos ni de las chicas. Debemos tener en cuenta que el alumnado estudiado pertenece en el primer momento a primero y segundo grado de ESO, mientras, en el segundo, a tercero y cuarto grado, por lo que este estancamiento puede estar relacionado con el crecimiento (Marques, 2010; Mourelle, 2014).

Dentro de la práctica en las actividades organizadas analizamos las AF de club o colectivo fuera (club) y dentro de la escuela (extraescolares) con una duración de más de 30 minutos. En ellas comprobamos que en las actividades de club existen dos grandes grupos en el total de los centros: un 25\% de alumnado que las realiza de dos a tres veces por semana (chicos $=30 \%$ y chicas $=20 \%$ ), con una duración de dos a tres horas (chicos $=46 \%$ y chicas $=39 \%)$ y un $46 \%$ que no las practica nunca (chicos $=36 \%$ y chicas $=57 \%)$.

La segunda de las prácticas organizadas que analizamos fueron las actividades extraescolares. En el to- tal de los centros no son practicadas por el $61 \%$ del alumnado, siendo mayor el porcentaje de chicas que no las realizan nunca (68\%) que de chicos $(54 \%)$; del escaso porcentaje que sí las realizan, las chicas suelen dedicarle una hora $(38 \%)$ y los chicos de dos a tres $(34 \%)$.

En correspondencia con nuestros datos, Marques (2010) da buena cuenta de cómo, aunque la mayor parte de las escuelas disponen de actividades extraescolares, desgraciadamente tan solo el $17 \%$ del alumnado las practica. Además, observando los datos generales de las actividades organizadas, vemos datos similares a los nuestros, como es el caso del estudio de Hábitos de la Población Escolar Española (Consejo Superior de Deportes Español, 2015), que encuentra que el $64 \%$ de los chicos adolescentes practican AF organizada una vez a la semana, pero este porcentaje desciende al 35\% en las chicas y al $30 \%$ en los adolescentes que las realizan tres veces por semana.

$\mathrm{Al}$ observar las diferencias entre momentos, sólo se encuentran diferencias significativas en la frecuencia de práctica de club de los chicos $(p=.003$ en todos los centros), correspondientes al descenso en los que realizaban de cuatro a seis veces a la semana y al aumento de los que nunca hacían.

Para finalizar, queremos recordar que el tiempo transcurrido entre la primera toma de datos y la segunda atiende al periodo comprendido entre principios de un curso escolar y finales del segundo curso académico. Este periodo, que en la mayoría de los centros era el tiempo que se llevaba aplicando el PDC, sin duda es escaso para llevar a cabo un cambio de hábitos y estilos de vida, por lo que creemos que los cambios, aunque escasos, deben ser vistos con optimismo y deben seguir alentando al profesorado y a las direcciones de los centros a apostar por estos proyectos de innovación educativa.

\section{Referencias}

Ballesteros-Arribas, J. M., Dal-Re Saavedra, M., Pérez-Farinós, N., \& Villar-Villalba, C. (2007). La estrategia para la nutrición, actividad física y preven- 
ción de la obesidad (Estrategia NAOS). Revista Española de Salud Pública, 81(5), 443-449.

https://doi.org/10.1590/s1135-572720070005 00002

Bermúdez, C. (2015). Implicaciones educativas de los beneficios de la actividad físico-deportiva. e-Motion: Revista de Educación, Motricidad e Investigación, (5), 3-16. https://doi.org/10.33776/remo.v0i5.2638

Carreiro da Costa, F., \& Marques, A. (2011). Promoting active and healthy lifestyle at school: Views of students, teachers, and parents in Portugal. In K. Hardman \& K. Green (Eds.). Contemporary issues in Physical Education: International Perspectives (pp.249-268). Meyer \& Meyer Sport.

Consejo Superior de Deportes Español (2015). Estudio los Hábitos Deportivos de la Población Escolar en España. Presidencia del Gobierno.

https://bit.ly/2PueuJj

Corbin, Ch. (2014). Fitness for Life Physical Activity Pyramid for Teens Poster. Human Kinetics.

Crawford, M. \& Unger, R. (2004). Women and Gender: A feminist psychology. McGraw-Hill.

Fundación para la Investigación Nutricional (2016). Informe 2016: Actividad Física en niños y adolescentes en España. Gobierno de España.

https://bit.ly/33USR9a

Grupo Colaborativo de la Sociedad Española de Nutrición Comunitaria (SENC) (2016). Guías alimentarias para la población española (SENC, diciembre, 2016); la nueva pirámide de la alimentación saludable. Nutrición Hospitalaria, 33 (Suplemento 8), 1-48. https://bit.ly/36bVsx5

Hallal, P. C., Andersen, L. B., Bull, F. C., Guthold, R., Haskell, W., \& Ekelund, U. (2012). Global physical activity levels: surveillance progress, pitfalls, and prospects. The Lancet, 380(9838), 247-257. https://doi.org/10.1016/s0140-6736(12)60646-1

Jago, R., McMurray, R. G., Bassin, S., Pyle, L., Bruecker, S., Jakicic, J. M., Moe, E., Murray, Y., \& Volpe, S. L. (2009). Modifying Middle School Physical Education: Piloting Strategies to Increase Phy- sical Activity. Pediatric Exercise Science, 21(2), 171185. https://doi.org/10.1123/pes.21.2.171

Martínez-Baena, A. C. (2012). Factores influyentes de la actividad físico-deportiva de adolescentes españoles: opiniones sobre el papel de la escuela (Tesis doctoral). Universidad de Granada, Granada. https://bit.ly/2PksbcG

Martínez-López, E. J., Lozano Fernández, M. L., Zagalaz Sánchez, M. L., \& Romero Granados, S. (2009). Valoración y autoconcepto del alumnado con sobrepeso. Influencia de la escuela, actitudes sedentarias y de actividad física. RICYDE. Revista Internacional de Ciencias del Deporte, 5(17), 44-59. https://doi.org/10.5232/ricyde2009.01704

Marques, A., \& Carreiro da Costa, F. (2010). A escola, a educação física e a promoção de estilos de vida saudáveis. Estudo de um caso. Novas Ediçóes Acadêmicas.

Marques, A., \& Carreiro da Costa, F. (2013). Levels of physical activity of urban adolescents according to age and gender. International Journal of Sports Science, 3(1), 23-27. https://bit.ly/2qqgSY8

Mayor Villalaín, A. (2018). Los proyectos deportivos como medio de promoción de actividad física en los centros escolares gallegos: tratamiento desde una perspectiva de género (Tesis doctoral). Universidade da Coruña, Coruña. https://bit.ly/2DUCn6p

Melnyk, B. M., Small, L., Morrison-Beedy, D., Strasser, A., Spath, L., Kreipe, R., Crean, H., Jacobson, D., Kelly, S., \& O'Haver, J. (2007). The COPE Healthy Lifestyles TEEN Program: Feasibility, Preliminary Efficacy, \& Lessons Learned from an After School Group Intervention with Overweight Adolescents. Journal of Pediatric Health Care, 21(5), 315322. https://doi.org/10.1016/j.pedhc.2007.02.009

Martínez Gómez, David, \& Veiga Núñez, Oscar Luis (2008). Guia para una escuela activa y saludable. Orientaciones para los centros de Educación Primaria. Secretaría General Técnica. Centro de Publicaciones. Ministerio de Educación, Política Social y Deporte-Ministerio de Sanidad y Consumo.

http://bit.ly/2LWtfTb 


\section{MAYOR-VILLALAÍN • CANTERO-CASTRILLO • TOJA-REBOREDO • GONZÁLEZ-VALEIRO}

Papel de la escuela y los proyectos deportivos de centro en el fomento de la práctica...

Ministerio de Sanidad, Servicios Sociales e Igualdad (2015). Actividad Física para la Salud y Reducción del Sedentarismo. Recomendaciones para la población. Estrategia de Promoción de la Salud y Prevención en el SNS. Ministerio de Sanidad, Servicios Sociales e Igualdad-Ministerio de Educación, Cultura y Deporte. http://bit.ly/2snLFFE

Moreno, L., Gonzalez-Gross, M., Kersting, M., Molnár, D., De Henauw, S., Beghin, L., Sjöström, M., Hagströmer, M., Manios, Y., Gilbert, C., Ortega, F., Dallongeville, J., Arcella, D., Wärnberg, J., Hallberg, M., Fredriksson, H., Maes, L., Widhalm, K., Kafatos, \& Marcos, A. (2008). Assessing, understanding and modifying nutritional status, eating habits and physical activity in European adolescents: The HELENA (Healthy Lifestyle in Europe by $\mathrm{Nu}-$ trition in Adolescence) Study. Public Health Nutrition, 11(3), 288-299.

https://doi.org/10.1017/s1368980007000535

Morgan, C. F., McKenzie, T. L., Sallis, J. F., Broyles, S. L., Zive, M. M., \& Nader, P. R. (2003). Personal, Social, and Environmental Correlates of Physical Activity in a Bi-Ethnic Sample of Adolescents. Pediatric Exercise Science, 15(3), 288-301.

https://doi.org/ 10.1123/pes.15.3.288

Mourelle, M. (2014). Relación de los agentes sociales con el indice de práctica de actividad fisica de los escolares de 10 a 16 años de las urbes gallegas (Tesis doctoral). Universidade da Coruña, A Coruña.

https://bit.ly/2ECzjMr

Nuviala Nuviala, A., Tamayo Fajardo, J. A., Fernández Martínez, A., Pérez-Turpin, J. A., \& Nuviala Nuviala, R. (2011). Calidad del servicio deportivo en la edad escolar desde una doble perspectiva. Revista Internacional de Medicina y Ciencias de la Actividad Fisica y del Deporte, 11(42), 220-235. https://bit.ly/2rqOAwT

Organización Mundial de la Salud (2010). Global recommendations on physical activity for health. WHO Press, World Health Organization.

https://bit.ly/2sTTh2J

Organización Mundial de la Salud (2015). Pre- valence of insufficient physical activity among school going adolescents, ages 11-17 (crude estimates). https://bit.ly/2Ly9KAm

Pérez-López, I., \& Delgado-Fernández, M. (2007). Mejora de los conocimientos, procedimientos y actitudes del alumnado de secundaria tras un programa de intervención en Educación Física para la salud. Motricidad. European Journal of Human Movement, (18), 61-77. https://bit.ly/2DZBYQ2

Piéron, M., García Montes, M. E., \& Ruiz Juan, F. (2007). Algunos correlatos de la actividad físico-deportiva en una perspectiva de salud. Tándem: Didáctica de la Educación Física, (24), 9-24. https://bit.ly/38lNFyr

Piéron, M., García Montes, M. E., \& Ruiz Juan, F. (2008). La opinión del alumnado de enseñanza secundaria sobre las clases de educación física: un desafío para los profesores y los formadores. Fuentes: Revista de la Facultad de Ciencias de la Educación, (8), 159-175. https://bit.ly/36cFkeH

Real Decreto 1105/2014. Currículo básico de la Educación Secundaria Obligatoria y del Bachillerato. Ministerio de Educación, Cultura y Deporte. Reino de España, 26 de diciembre de 2014. https://bit.ly/353VeaW

Riddoch, C., Edwards, D., Page, A., Froberg, K., Anderssen, S. A., Wedderkopp, N., Brage, S., Cooper, A., Sardinha, L., Harro, M., Klasson-Heggebø, L., van Mechelen, W., Boreham, C., Ekelund, U., \& Andersen, L. B. (2005). The European Youth Heart Study-Cardiovascular Disease Risk Factors in Children: Rationale, Aims, Study Design, and Validation of Methods. Journal of Physical Activity and Health, 2(1), 115-129. https://doi.org/10.1123/jpah.2.1.115

Roberts, C., Freeman, J., Samdal, O., Schnohr, C. W., De Looze, M. E., Gabhainn, S. N., Iannotti, R., Rasmussen, M. (2009). The Health Behaviour in School-aged Children (HBSC) study: methodological developments and current tensions. International Journal of Public Health, 54(2), 140-150. https://doi.org/10.1007/s00038-009-5405-9 
Romero C., R. (2007). Delimitación del campo didáctico de la Educación Física y de su actividad científica. Profesorado. Revista de Curriculum y Formación del Profesorado, 11(2). https://bit.ly/2P0Yuyj

Ruiz-Pérez, L. M., Ramón-Otero, I., Palomo-Nieto, M., Ruiz-Amengual, A., \& Navia-Manzano, J. A. (2014). La intención de practicar en el futuro en escolares adolescentes. Kronos, 13(2). https://bit.ly/2rpVTVL

Sallis, J. F., Bull, F., Guthold, R., Heath, G. W., Inoue, S., Kelly, P., Oyeyemi, A., Pérez, L., Richards, J., \& Hallal, P. (2016). Progress in physical activity over the Olympic quadrennium. The Lancet, 388(10051), 1325-1336.

http://dx.doi.org/10.1016/S0140-6736(16)30581-5

St Leger, L., \& Young, I. M. (2009). Creating the document 'Promoting health in schools: from evidence to action'. Global Health Promotion, 16(4), 69-71. https://doi.org/10.1177/1757975909348138

Tremblay, M. S., Carson, V., Chaput, J.-P. ..., \& Zehr, L. (2016). Canadian 24-Hour Movement Guidelines for Children and Youth:An Integration of Physical Activity, Sedentary Behaviour, and Sleep. Applied Physiology, Nutrition, and Metabolism, 41(6), S311-S327.

https://doi.org/10.1139/apnm-2016-0151

Vázquez, B., Fernández, E., \& Ferro, S. (2000). Educación Física y género: Modelo para la observación y el análisis del comportamiento del alumnado y del profesorado. Gymnos.

Villard, L. C., Rydén, L., \& Ståhle, A. (2007). Predictors of healthy behaviours in Swedish school children. European Journal of Cardiovascular Prevention \& Rehabilitation, 14(3), 366-372.

https://doi.org/10.1097/01.hjr.0000224487.26092.a3

Mayor-Villalaín, A., Cantero-Castrillo, P., Toja-Reboredo, B., \& González-Valeiro, M. A. (2019). Papel de la escuela y los proyectos deportivos de centro en el fomento de la práctica de actividad física de los escolares. Revista Caribeña de Investigación Educativa (RECIE), 3(2), 56-72. https://doi.org/10.32541/recie.2019.v3i2.pp56-72 